\title{
FUZZY INCLUSION IN DATABASE AND INFORMATION RETRIEVAL QUERY INTERPRETATION
}

\author{
G. BORDOGNA, P. BOSC, G. PASI \\ CNRITIM (taly) and IRISA/ENSSAT (France)
}

Keywords: flexible queries, fuzzy sets, cardinality of a fuzzy set, fuzzy implication, document retrieval, division of relations.

Abstract. In this paper, a short review of the role of the inclusion operator in the interpretation of queries addressed to databases and Information Retrieval Systems (IRSs) is analyzed. Some properties and semantic aspects of various definitions of fuzzy. inclusion are discussed and applied to interpret queries in Data Base Management Systems and IRSs.

\section{INTRODUCTION}

In the area of information systems, a significant amount of activity aims at extending the functionalities provided by the systems. In this spirit, a thread of research has focused on the introduction of imprecision in information systems, both for representing imperfectly known data and for expressing imprecise queries. In this context, basic operations have been reconsidered, such as mechanisms for selecting the desired information (conditions and agregation operators in particular). However, it turns out that more complex operators have not received much attention although they are important. In this paper, the focus is put on the inclusion operation and its role in database management systems (DBMSs) and information retrieval systems (IRSs) is studied. The role of the inclusion between two regular sets in conventional systems will be first recalled and then the case of "extended" systems dealing with fuzzy sets will be considered. In DBMSs, the inclusion underlies the division of two relations, such as in the query "find the stores which have sold a given set of items" with a database describing products and sales. The extension of the division to fuzzy relations has received several interpretations and we will see that some of them are directly inspired from the inclusion between furzy sets. In IRS models, a document is retrieved as far as it "contains" a set of desired key-words and "does not contain" a set of excluded key-words. This view highlights the fact that the inclusion is a very natural notion in the context of information retrieval and we will see that, indeed, several $\mathbb{R}$ models call on an inclusion to define the matching mechanism between a document and a query.

The paper is organized as follows. Sectiou 2 is dedicated to a short review of the role of the inclusion operator in the interpretation of regular queries addressed to databases and IRSs. In section 3, the two principal approaches to the definition of the inclusion between fuzzy sets are presented: the first one is based on fuzzy implications whereas the other uses the notion of cardinality of fuzzy sets. Some properties and semantic aspects of these various definitions are discussed. In section 4, the connections between the inclusion (according to the definitions given in the previous section) and proposals suggested in the literature to interpret queries in DBMSs and IRSs are investigated.

"Permission to make digital/hard copy of all or part of this material without fee is granted provided that copies are not made or distributed for profit or commercial advantage, the ACM copyright/server notice, the title of the publication and its date appear, and notice is given that copying is by permission of the Association for Computing Machinery, Inc.(ACM). To copy otherwise, to republish, to post on servers or to redistribute to lists, requires prior specific permission and/or a fee."

๑ 1996 ACM 0-89791-820-7 9600023.50
In the conclusion, the main contributions of the paper are summarized and some trends for future work are pointed out.

\section{INCLUSION IN QUERY INTERPRETATION}

The notion of inclusion is present in a more or less implicit way both in database and information retrieval query languages.

Inclusion in database queries

If we consider a relational database system, the algebraic operator known as the relational division is based upon the inclusion between regular sets. In fact, if $R(A, B)$ is a binary relation and $S(A)$ is a unary relation, the division $R(A+A] S$ of $R$ by $S$ aims at determining the $B$-values in $R$ which are connected with all the A-values in S:

$b \in R[A \div A] S \Leftrightarrow \forall a, a \in S \Rightarrow(a, b) \in R$

For example, if $S$ (item) represents the items whose price is over $\$ 15$ and R(store, item) contains tuples of the form $\langle s, i\rangle$ where the item $i$ has been ordered by the store $s$ in a quantity under 50, the query looking for the stores having ordered less than 50 pieces of all items whose price is over $\$ 15$ is the division R[item+ item]S.

Another (equivalent) view of this operator is:

$b \in R[A \div A] S \Leftrightarrow S \subseteq R_{b}[A]$

where $R_{b}[A]$ denotes the set of $A$-values associated with the value $b$ in relation $R$ (i.e; if tuple $<a l, b>$ belongs to $R$, al belongs to $R_{b}[A]$ ).

One may now imagine to change the previous query into: "find the stores having ordered a low number of pieces of all medium-priced items", which calls on a division involving fuzzy relations, $\mathrm{R}^{\prime}$ (resp. $\mathrm{S}^{\prime}$ ) expressing the extent to which the number of pieces ordered is low (resp. the price of an item is medium). However, it is important to remark that one may have sliglitly different interpretations of this operation, especially depending on the intended interaction between the weights associated with the tuples in relations $\mathrm{R}$ and $\mathrm{S}$. Lastly, although of great interest, the case where the universal quantifier is weakened, is not discussed here.

\section{Inclusion in information retrieval}

In the literature several models have been proposed to define information retrieval systems; among these, the one which constitutes the basis of the majority of commercial systems is the Boolean model. Here, $\mathbb{R}$ is conceived as a crisp matching between the representation of documents and that of the query. Documents are represented as sets of terms and queries are Boolean expressions on terms or negated terms. Any Boolean query can be translated in disjunctive normal form. Each disjunct identifies a subquery $q_{i}$, which demands for documents including not negated terms and excluding negated ones. The whole query retrieves then the union of documents 
retrieved by each disjunct. This makes clear the role played by the inclusion operation in the Boolean matching.

This basic model of $\mathbb{I R}$ has been extended to the main aim of providing a flexible matching mechanism able to evaluate the degree of relevance or satisfaction of each retrieved document with respect to the query. These models are based on two main ideas: the association of a weight with both each term in the representation of documents (index tenn weights) and each term in the query (query term weight).

Index term weights express the significance of terms in representing the document contents, while query term weights indicate the importance that terms should have in the desired documents.

The introduction of the index term weight makes it possible to represent a document as a fuzzy set of terms [Wall 79, Buel 81, Buel 82]: $R(d)=\left\{\mu_{d}(t) / t, t \in T\right\}$ in which $\mu_{d}(t)=F(d, t)$.

In the case of IRSs with weighted Boolean queries, the matching mechanism can be seen as applying two conditions:

1. selection condition: documents including not negated tenns and excluding negated ones are considered;

2. ordering condition: documents selected by the first condition are ranked according to their degree of satisfaction, called Retrieval Status Value (RSV), of a function $E^{*}: D \times Q \rightarrow[0,1] . E^{*}$ evaluates the $A N D, O R$ and NOT in the query as the minimum, the maximum and the complement respectively, and each pair term-weight $(\langle t, w\rangle)$ by applying a function $E$ depending on the query weights' semantics.

In the following, the main semantics for query term weights defined in the literature are introduced by their corresponding E function.

The "relative importance" semantics defines query weights as measures of the "relative importance" of each term with respect to the others in the query [Book 80, Yage 87]. They demand for retrieval results conditioned more heavily by the most heavily weighted terms. In a first model [Book 80], this semantics has been formalized within fuzzy set theory depending on the operators connecting the query terms: it is a product when the terms are linked by the OR operator:

$$
E(d,<t, w)=w * F(d, t),
$$

and a ratio when the terms are linked by the AND operator:

$$
E\left(d,<t, w>=\left\{\begin{array}{l}
F(d, t) / w \text { for } F(d, t)<w \\
1 \text { otherwise. }
\end{array}\right.\right.
$$

A second formalization of query weights as "relative" importance weights has been proposed in [Yage 87]; also in this model two definitions of $\mathrm{E}$ are proposed for conjunctive and disjunctive queries; in conjunctive queries the $\mathrm{E}$ function is defined as:

$$
E(d,<t, w>)=\max [(1-w), F(d, t)]
$$

In case of disjunctive queries $\mathrm{E}$ is defined as:

$$
E(d,<t, w)=\min [w, F(d, t)] .
$$

Other authors proposed a "threshold" semantics for query weights [Rade 79, Buel $81 \mathrm{a}$, Buel $81 \mathrm{~b}$ ]. By specifying thresholds the user is asking to see all documents "sufficiently about" a topic. The lower the threshold, the greater the number of documents retrieved. The threshold allows to define a point of discrimination between under and oversatisfaction (with respect to the threshold). The simplest formalization of threshold weights has been suggested in [Rade 79] where the E function is defined as a crisp threshold:

$$
E\left(d, 4, w>=\left\{\begin{array}{l}
0 \text { for } F(d, t) \leq w \\
F(d, t) \text { otherwise. }
\end{array}\right.\right.
$$

In this case, the threshold defines the minimally acceptable document. Due to its discontinuity this definition may cause the abrupt variation of the number of documents retrieved even for small changes in the query weights. This led Buell and Kraft to define continuous thresholding functions [Buel 81a, Buel81b]. These authors have suggested the following definition for $\mathrm{E}$ :

$E(d,<t, w>)=\left\{\begin{array}{c}P(w) * \frac{F(d, t)}{w} \text { for } F(d, t)<w \\ P(w)+Q(w) * \frac{(F(d, t)-w)}{(1-w)} \text { otherwise }\end{array}\right.$

in which: $P(w)=\frac{1+w}{2} \quad Q(w)=\frac{1 \cdot w^{2}}{4}$

For $F<w$, the $E$ function measures the closeness of $F$ to $w$, for $F^{3} w, E$ expresses the degree of oversatisfaction with respect to $w$, and undersatisfac-tion with respect to 1 .

Since extended Boolean models have been defined within fuzzy set theory, the aim of this paper is to investigate if the whole query evaluation can be regarded in terms of a fuzzy inclusion. In this case, the RSV could be interpreted as a degree of inclusion of the query in the documents. This analysis is dealt with in the last section.

\section{POSSTBLE DERINITIONS FOR THE FUTZY INCLUSION}

Two main approaches are possible for extending the regular inclusion: fuzzy implications and cardinalities.

\section{Degree of inclusion and fuzxy implications}

A first view of the inclusion consists in defining a degree of inclusion $\mathrm{d}(\mathrm{E} \subseteq \mathrm{F})$ using a fuzzy implication [1]: $\mathrm{E} \subseteq \mathrm{F} \Leftrightarrow$ $\forall x((x \in E) \Rightarrow(x \in F)) \Leftrightarrow \forall x\left(\mu_{\mathrm{E}}(x) \rightarrow \mu_{F}(x)\right)$, and then, we obtain the indice:

$d(E \subseteq F)=\min _{x}\left(\mu_{E}(x) \rightarrow \mu_{F}(x)\right)$

Two slightly different interpretations may be distinguished depending on the nature of the interaction of the degrees in the two sets. In the first case, the degree $\mu_{F}(x)$ is seen as a threshold and the complete satisfaction requires that this threshold is attained by $\mu_{R}(x)$ for each value $x$ of $X$. When it is not the case, a penalty is applied. This behavior is achieved by implications of the type:

$u \rightarrow v=1$ if $v \geq u, t$ otherwise.

The following implications:

Rescher-Gaines: $u \rightarrow_{R G} v=1$ if $u \geq v, 0$ otherwise

Gódel: $u \rightarrow_{G d} v=1$ if $u \geq v, v$ otherwise

Godel reciprocal: $u \rightarrow_{G d R} v=1$ if $u \geq v, 1-u$ otherwise

Goguen: $u \rightarrow{ }_{G g} v=1$ if $u \geq v, v / u$ otherwise

Lukasiewicz: $u \rightarrow_{L} v=l$ if $u \geq v, l+v-u$ otherwise

comply with this pattern and thus are potential candidates for this kind of interpretation of the extended inclusion. However, from a user point of view, these implications correspond to 
different semantics when the strict inclusion does not hold. (4) is not really interesting since it involves no graduality (and in fact it corresponds exactly to Zadeh's inclusion). With Godel implication, when the threshold is not attained, the result is somewhat "absolute" since it does not depend at all on $\mu_{f}(x)$, but a purely ordinal scale is sufficient. If we are provided with a richer scale, Goguen implication may be more convenient since the result reflects the ratio between $\mu_{E}(x)$ and $\mu_{F}(x)$. It must be noticed that in both cases, any element in $F$ must be in $\mathrm{E}$, otherwise the result of the inclusion is null. This fact is obviated if Godel reciprocal or Lukasiewicz implications are used. However, in both cases, if $F$ is not normalized, its degree of inclusion in the empty set is not null, which may be troublesome. The following orderings hold anong the implications:

$u \rightarrow_{R G} v \geq u \rightarrow_{G d} v \geq u \rightarrow_{G g} v \geq u \rightarrow_{L} v$

$u \rightarrow_{R G} v \geq u \rightarrow_{G d R} v \geq u \rightarrow_{L} v$.

Of course, these orderings entail orderings over the degrees of inclusion based on the respective implications.

In the second cae, $\mu_{F}(x)$ defines the importance of $x$ (and then the degree $\mu_{E}(x)$ is modulated). In the logical framework imposed by an implication, the underlying notion is that of a guaranteed satisfaction when this importance is under 1 . When $\mu_{F}(x)<1$, i.e., the element $x$ is not completely important and it can be forgotten to some extent. The complete satisfaction requires that $\mu_{\mathrm{E}}(\mathrm{x})$ equals 1 for each value $\mathrm{x}$ of $\mathrm{F}$ whatever its importance. This behavior leads to define the division by using Dienes implication $u \rightarrow_{K D} v=\max (1-u, v)$ and to adopt:

$$
\begin{aligned}
d(E \subseteq F)= & \min _{x \in X} \mu_{F}(x) \rightarrow K D \mu_{E}(x)= \\
& \min x \in x \max \left(1-\mu_{F}(x), \mu_{E}(x)\right)
\end{aligned}
$$

where $F$ is a nonnalized fuzzy relation $\left(\exists u, \mu_{F}(u)=1\right)$ in order to have an appropriate scaling of the levels of importance. Here again, ordinal scales on $E$ anf $F$ are sufficient.

\section{Degree of inclusion and cardinality}

Another way to define a degree of inclusion [2] is to consider: $E \subseteq F \Leftrightarrow(\operatorname{Card}(E \cap F)=\operatorname{Card}(E))$ and then the degree is given by:

$$
d(A \subseteq B)=\frac{\sum \operatorname{Count}(A \cap B)}{\sum \operatorname{Count}(A)}=\frac{\sum_{x \in X} o p_{1}\left(\mu_{A}(x), \mu_{B}(x)\right)}{\sum_{x \in X} \mu_{A}(x)}
$$

where opl is a triangular norm.

Here again, two cases may be distinguished depending on the role assumed by the degrees in $F$. If the degrees $\mu_{F}(x)$ act as thresholds on the values $\mu_{E}(x)$, the choice of the norm opl in formula 10 is the minimum.

On the contrary, if $\mu_{F}(x)$ is an importance assigned to the element $x$, the norm opl is the product; thus formula (10) becomes a weighted average.

\section{Comments and example}

The approach based on fuzzy inplications is logical and conjunctive and an "absorption effect" occurs: the inclusion only retains the smallest degree of inplication between $F$ and E. This phenomenon disappears with the second vision which is numerical and compensatory, where all the values
$\operatorname{opl}\left(\mu_{E}(x), \mu_{F}(x)\right)$. In each case, the $F$-grades can express either a Uureshold or an importance.

Example. Let us consider the following fuzzy sets:

$E=\{1 / \times 1, .4 / \times 2, .6 / \times 4\}, F=\{.7 / \times 1, .2 / \times 2, .3 / \times 3, .8 / \times 4\}$.

Depending on the interpretation chosen (implication in (3), tnonn in (10)), the degree of inclusion of $E$ in $F$ is given hereafter:

\begin{tabular}{|l|l|l|}
\cline { 2 - 3 } \multicolumn{1}{c|}{} & \multicolumn{1}{c|}{ implication } & \multicolumn{1}{c|}{ cardinality } \\
\hline importance & Kleene-Dienes: .6 & product: .63 \\
threshold & $\begin{array}{l}\text { Godel: .2 } \\
\text { Godel recip.: 0 } \\
\text { Goguen: .5 } \\
\text { Lukasiewicz: .7 }\end{array}$ & min: .75 \\
\hline
\end{tabular}

\section{FUZZY INCLUSION AS A BASIS OF RETRIEVAL IN DATABASES} AND INFORMATION RETRIEVAL SYSTLMS

\section{Fuzzy inclusion in flexible database queries}

Let us consider the division of two furzy relations $R(A, B)$ and $S(A)$ whose tuples are weighted by a number between 0 and 1 . The extension of the division to fuzzy sets defines naturally the result of the division as a fizzy set. Then, a natural extension stems from (2) where the usual set inclusion operator is changed into a degree of inclusion d defined as:

$\mu_{R(A+A] S}(b)=d\left(S \subseteq R_{b}(a)\right)$

where $R_{b}(a)$ is the fuzzy set defined by $\{\langle i / a\rangle \mid\langle\mu /(a, b)\rangle \in$ $R$ \}. If the inclusion is interpreted by means of a fuzzy implication $(\rightarrow)$ such as in formulae $(4)-(8)$, the general form of the expression becomes:

$\mu_{R[A+A] S}(b)=\min , \in s \mu_{S}(a) \rightarrow \mu_{R}(a, b)$

This operation is illustrated in the query: "find the stores having ordered a low number of pieces of all medium-priced items" addressed to a relational database composed of two regular relations ORDERS(store, item, quantity) and PRODUCT(item, price). This query is a flexible one since, due to the use of gradual predicates, one attempts to retrieve stores which are more or less acceptable rather than "completely satisfactory". By analogy with a query using only Boolean predicates (< 10 rather than "low number" and price between $\$ 10$ and \$25 rather than "medium-priced"), the interpretation of this query may be based on the division L-ORDERS[item * item]MP-PRODUCTS of the two fuzzy relations $L$ ORDERS(store, item) and MP-PRODUCTS(item) whose grades express the extent to which the quantity ordered by a store for an item is "low" and the price of the item is "medium". The extension of the division of relations to fuzzy relations has been studied by some researchers and their proposals are now reviewed to assess their relationship with fuzzy inclusions.

For N. Mouaddib [Moua 93], the division of two relations R(A, $B$ ) and $S(A)$ is defined by:

$\mu_{R}[A+A] S(b)=\min _{\mathbf{a} \in S} \min \left(\mu_{R}(a, b), \mu_{S}(a)\right)$

In formula (11), the universal quantifier is interpreted as the generalized conjunction ( $\mathrm{min}$ ), but the interaction between relations $\mathrm{R}$ and $\mathrm{S}$ is not modeled by an implication. 
In their generalization of the relational algebra, $M$. Umano and $S$. Fukami [Uman 94] suggest $\mu_{R}[A+A] s(b)$ to be:

$\min _{a} \in S \min \left(\mu_{R}(a, b), \mu_{S}(a), \mu_{S}(a) \rightarrow_{R G} \mu_{R}(a, b)\right)$

where $\rightarrow_{\mathrm{RG}}$ is Rescher-Gaines inplication. In formula 14, although a fuzzy implication is used, the formula does not correspond exactly to the pattern given in fornula 12 .

The next proposition comes from R. Yager [Yager 91] who has defined a division operator where the universal quantifier is weakened (for instance into "most"). When the universal quantifier is used, his definition is:

$\mu_{R}[A+A] S(b)=\min _{w \in S} \max \left(1-\mu_{S}(a), \mu_{R}(a, b)\right)$

Formula (15) fits the pattern proposed in formula 12 by choosing Kleene-Dienes implication. According to what has been discussed regarding the semantics of the implications, this proposal assumes that the weight tied to an element $a$ in relation $\mathrm{S}$ defines the importance of a. This approach is also advocated in [Cube 94].

The last proposition has been initially presented in [Dubo 94 , Bosc 95a] and it is has been enlarged in [Bosc 95b]. Basically, the idea is to allow for different interpretations of the division by choosing an inclusion operator according to formula 13 , based either on an implication (formulae 5 - 9) or on cardinalities (formula 10 with min or product). In addition to that, syntactic features of a query language called SQLf [Bosc 95c] are proposed to express queries involving fuzzy divisions. In this framework, a specific construct (the containment) is added to the language so as to support the division in the following way:

select $\mathrm{B}$ from $\mathrm{R}$ where $\mathrm{f} \mathrm{R}$ group by $\mathrm{B}$

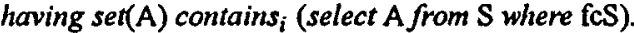

The interpretation of this sentence (which corresponds to $S \subseteq$ $\left.R_{b}[A]\right)$ is the following: i) group all the tuples having a same $B$-value and compute their membership degree with respect to the fuzzy condition $\mathrm{fcR}, \mathrm{ii}$ ) compute the membership degree of S-tuples with respect to fcS, iii) for each group $g$ of $R$-tuples, compute the degree of inclusion (according to contains $\mathrm{s}_{\mathrm{i}}$ ) of the A-values of $S$ in the A-values in $\mathrm{g}$.

Example. The SQLf expression of the query: "find the stores having ordered a low number of pieces of all medium-priced items" is:

select store from ORDERS where quantity $=$ "low'

group by store having set(item) contains $s_{G d}$

(select item from PRODUCT where price = 'medium'))

The key-word "contains $\mathrm{Gd}$ " stands for Godel implication and this choice means that the interpretation of the query is: the more medium the price of an item, the lower the quantity ordered by a store should be. With "contains $\mathrm{KD}$ " (KleeneDienes implication), the meaning would be: the more medium the price of an item, the more important the fact that the quantity ordered by a store is low.

\section{Fuzzy inclusion and document retrieval}

The purpose of this section is establish whether the retrieval applied by extended Boolean models described in section 2. can be formalized in terms of an inclusion between fuzzy sets. For the sake of simplicity, we consider in this context only conjunctive queries without negated terms.

The retrieval operation activated by a conjunctive query can be synthesized through the following sentence: "find documents which satisfy all the requirements on terms in the query. The retrieval can be described by an operation on the fuzzy relation $\mathrm{R}$ on documents and terms (where the membership value of a pair $\langle d, t\rangle$ is the significance degree $F(d, t)$ ), and $S$, where the membership value is the query term weight $w$ :

$R=\left\{\mu_{R}(d, t) /(d, t) \mid d \in D\right.$ and $\left.t \in T\right\}$ in which $\mu_{R}(d, t)=F(d, t)$ $S=\left\{\mu_{S}(t) / t \mid t \in Q\right\}$ in which $\mu_{S}(t)=w$.

The retrieval activity is aimed at establishing a connection between the values $\mu_{S}(t)$ and $\mu_{R}(d, t)$, to evaluate how well the value $\mu_{R}(d, t)$ satisfies the requirement $\mu_{S}(t)$; as seen in the previous section the type of connection depends on the adopted retrieval function $\mathrm{E}$.

Let us remind how the the division of fuzzy relations $R \div S$ can be used to model information retrieval mechanisms:

$$
\mu_{R+S}(d)=\min t \in S\left(\mu_{S}(t) \rightarrow \mu_{R}(d, t)\right) .
$$

Here, the fuzzy set resulting from the division $\left\{R S V_{i} / d_{i}, d_{i} \in\right.$ $D$ is defined on the universe $D$ of documents, and the membership value associated with a document, i.e. the RSV, is the degree of the inclusion $\mu_{\mathrm{R}+\mathrm{S}}(\mathrm{d})$.

As seen in section 2., the RSV of a document is computed by the function $\mathrm{E}^{*}$ evaluating a query. When the query consists of a disjunct without negated terms $E^{*}$ can be defined in terms of a function $E$ evaluating a pair $\langle t, w>$ as follows:

$$
E^{*}(d, q)=\min _{i=1, \ldots, n} E\left(d, \psi_{i}, w_{i}>\right)
$$

We can say that $E^{*}(d, q)=\mu_{R+s}(d)$ if $E(d, \varangle, w>)$ can be defined in terms of a fuzzy implication.

In the next subsections, the previous $\mathrm{IR}$ models are analyzed with respect to fuzzy implications.

In the model with a fuzzy document representation, while the relation between terms and documents is defined in $[0,1]$, the implicit query weights are equal to 1 for all the terms not negated in the query. Thus the query $q$ may be seen as the specification of a limit case of a fuzzy set, defined on the set of query terms: $S=\left\{\mu_{S}(t) / t \mid t \in q\right\}$ with $\mu_{S}(t)=1$. The $E^{*}$ function for a conjunctive query is defined as $E^{*}(d, q)=\min _{t \in}$ $q(F(d, t))$. Function $E$ evaluating a single term in the query can then be defined as any fuzzy implication:

$$
E(d,<t, l>)=\mu_{S}(t) \rightarrow \mu_{R}(d, t)
$$

which produces $\mu_{R}(d, t)(F(d, t))$ when $\mu_{s}(t)=1$ (for example Godel, Lukasiewicz, Goguen, Kleene-Dienes, etc.). In this case, all the terms in the query have an implicit weight 1; the semantics of these weights can be interpreted as an importance (all terms have the maximum importance) as well as a threshold (the maximum satisfaction is obtained when $\mu_{R}(d, t)$ is 1 ).

In the first formalization of the $\mathbb{R}$ model based on the relative semantics due to Bookstein, the E function is defined exactly as Goguen implication (7):

$$
E(d,<t, w>)=\left\{\begin{array}{l}
1 \text { if } w \leq F(d, t) \\
\frac{F(d, t)}{w} \text { otherwise }
\end{array}\right.
$$


It can be noticed that the weights play truly a role of importances only when they are not reached by the $F(d, t) s$; consequently this behavior can also be interpreted as a threshold according to (7).

In the formalization due to Yager, the $E$ function is KleeneDienes implication (9) extending the classical implication (w $\left.\rightarrow_{\mathrm{XD}} \mathrm{F}(\mathrm{d}, \mathrm{t})=\neg \mathrm{w} \vee \mathrm{F}(\mathrm{d}, \mathrm{t})\right)$

$$
E(d,<t, w)=\max (1-w, F(d, t))
$$

in which the weight $w$ is properly an importance.

The threshold semantics for query weights is in its nature a twofold semantics. When associating a threshold weight to a query term a user is introducing a minimum acceptance level of the $F(d, t)$ values. The association of a value $w$ to a query term implies then a crisp classification of the $F(d, t) s$ in documents to be analyzed: $F(d, t)$ values under and over $w$. The behaviour of function $E$ for values belonging to one of the two classes is distinct, and it is modelled by two different functions.

For $F(d, t) s$ over $w$ the requirement is that the higher the significance of the term in a document $d$, the higher must be the RSV, i.e. the value $E(d, \varangle, w>)$. The maximum RSV is obtained when $F(d, t)=1$, i.e. when the implicit threshold 1 is reached. For $F(d, t) s$ under $w$, the RSV must be penalized: in the Bookstein definition, no satisfaction at all is obtained when $F(d, t)<w(E(d,<t, w)=0)$, while in the Buell \& Kraft definition a degree of satisfaction is obtained, which is a penalization of $F(d, t)$ depending on $w(E(d, \varangle, w)=F(d, t) / w)$. In this section, a relationship is pointed out between the threshold functions and fuzzy implications. At a first sight, one may observe both a similarity and a difference between a fuzzy implication and a threshold function. In a threshold function, $F(d, t)$ is compared with $w$ like in the implication $w \rightarrow F(d, t)$ (in particular both are monotonically increasing on $F(d, t)$ and decreasing on $w$ ), but in case of a fuzzy implication a complete satisfaction is obtained when $F(d, t)$ is over (or equal to) $w$, whereas this is not the case of the threshold functions defined in [Rade 79, Buel 81a]. These functions express a stricter constraint than that imposed by a fuzzy implication. It follows that these threshold functions are not directly expressible as fuzzy implications and our goal is to model them as a "modified" fuzzy implication.

Considering Radecki's formalization of threshold semantics, the following expression may be obtained:

$$
E(d,<t, w)=\left(w \rightarrow_{R G} F(d, t)\right) * F(d, t)
$$

where $\rightarrow_{R G}$ is Rescher-Gaines implication (formula 4).

Considering Buell and Kraft definition, the following reformulation of the $\mathrm{E}$ function can be obtained:

$$
\begin{aligned}
E(d,<t, w)= & \left(w \rightarrow_{G g} F(d, t)\right) * \\
& \left(P(w)+Q(w) * \frac{\max (F(d, t)-w, 0)}{1-w}\right.
\end{aligned}
$$

where $\rightarrow_{\mathrm{Gg}}$ is Goguen implication (7). In both cases function $\mathrm{E}$ is defined by modifying a "threshold-based" implication, so as to obtain the highest satisfaction only when $F(d, t)=1$. In the other cases of threshold oversatisfaction, a penalty is applied too; this is a slightly different interpretation of the notion of a threshold.

\section{Conclusion}

In this paper the role played by the inclusion operation in both the division of fuzzy relations in DBMSs and in weighted query evaluations in extended Boolean luformation Retrieval is

investigated. Some fuzzy approaches presented in the literature are refonmulated in the unified framework of fuzzy inclusion. Future developments of this work will concern two points:

i) the weakening of the universal quantifier implied in the division of fuzzy relations and ii) the consideration of more general queries (not only conjunctive) in IRSs.

Authors' addresses:

G. Bordogna and G. Pasi

CNR/TTIM

Via Ampere 56

20131 MILANO

ITALY

tel: 39.2 .70643257

fax: 39.2 .7064 .3292

e-mail: fuzzin@itim.mi.crr.it

\author{
P. Bosc \\ IRISA/ENSSAT \\ 6, nue de Kérampont \\ BP 447 \\ 22305 LANNION CédeX \\ FRANCE \\ tel: 33.96 .46 .66 .45 \\ fax: 33.96.37.01.99 \\ o-mail: boso@enssat.fr
}

\section{REFERENCES}

[Book 80] Bookstein A. (1980). Fuzzy requests: an approach to weighted Boolean searches. Joumal of the American Society for Information Science, 31, 240-247.

[Bosc 95a] Bosc P., Dubois, D., Pivert, O., Prade H. (1995).

Fuzzy division for regular relational databases, 4th Int. IEEE Conference on Fuzzy Systems (FUZZ-IEEE/IFES'95), Yokohama (Japan), March 1995, 729-734.

[Bosc 95b] Bosc P. (1995). Some views of the division of fuzzy relations, Sth Int. Workshop on Current lssues on Fuzzy Technologies (CIFT'95), Trento (Italy), 14-22

[Bosc 95c] Bosc P, Pivert O. (1995). SQLf: a relational database language for fuzzy querying, IEEE Transactions on Fuzzy Systems, 3(1), 1-17.

[Buel 82] Buell D.A. (1982). An analysis of some fuzzy subset applications to information retrieval systems. Fuzzy Sets and Systems, 7, 35-42.

[Buel 81a] Buell D.A., and Kraft D.H. (1981a). Threshold values and Boolean retrieval systems. Information Processing \& Management, 17, 127-136.

[Buel 81b] Buell D.A., and Kraft, D.H. (1981b). A model for a weighted retrieval system. Joumal of the American Society for Information Science, 32, 211-216.

[Cube 94] Cubero J.C., Medina J.M., Pons O., Vila M.A. (1994). The generalized selection: an alternative way for the quotient operations in fuzzy relational databases. 5 th Conference on Information Processing and Management of Uncertainty (IPMU'94), Paris (F), 23-30.

[Dubo 94] Dubois D., Prade H. (1994). Quotient operators in fuzzy relational databases, 2nd European Congress on Fuzzy and Intelligent Techniques (EUFT'94), Aachen (G), 357-360.

[Rade 79] Radecki T. (1979). Fuzzy set theoretical approach to document retrieval. Information Processing \& Management, $15,247-260$.

[Moua 93] Mouaddib N. (1993). The nuanced relational division, 2nd IEEE Intermational Conference on Fuzzy Systems (FUZZ-IEEE'93), San Francisco (USA), 1419-1424.

[Uman 94] Umano M., Fukami S. (1994). Fuzzy relational algebra for possibility-distribution-furzy-relational model of fuzzy data, Joumal of Intelligent Information Systems, 3(1), 7 28.

[Wall 79] Waller W.G., and Kraft D.H. (1979). A mathematical model of a weighted Boolean retrieval system. Information Processing \& Management, 15, 235-245.

[Yage 87] Yager R. R. (1987). A note on weighted queries in information retrieval systems, Joumal of the American Society for Infomation Science, 38, 23-24.

[Yage 91]Yager R.R. (1991).Furzy quotient operators for fuzzy relational databases, Intemational Fuzzy Engineering Symposium, Yokohama (Japan), 289-296. 